\title{
The 3D flow of a liquid through a porous medium with absorbing and swelling granules
}

\author{
ANTONIO FASANO \\ Dipartimento di Matematica “U. Dini”, Viale Morgagni 67/A, 50134 Firenze, Italy \\ AND \\ ANDRO MIKELIĆ ${ }^{\dagger}$ \\ UFR Mathématiques, Université Claude Bernard Lyon 1, Bâtiment 101, 43 Bd du 11 novembre \\ 1918, 69622 Villeurbanne Cedex, France
}

[Received 19 July 2001 and in revised form 3 March 2002]

\begin{abstract}
We study the flow of an incompressible fluid through a porous medium with hydrophile granules. The system is schematized as a periodic array of cubic cells, each containing one spherical swelling granule. The physical situation is such that the size of the granules is of the same order of the size of the cells and much larger than the microscopic constituents of the porous matrix. The porosity at each point of a cell is defined according to the size of the granules located at the cell vertices. The swelling of each granule is governed by a kinetic law involving the average moisture content of the medium over the granule surface.

The notion of weak solution is introduced and we prove the existence of such solution using backward time differences. The discretized problem is studied in detail and appropriate a priori estimates are obtained. Passing to the limit requires a precise analysis of the convergence in the geometry evolving with the solution.
\end{abstract}

Keywords: porous media with swelling granules; diapers; absorption; degenerate parabolic equation in a moving geometry.

\section{Introduction}

The flow of a liquid through a diaper is a complex phenomenon because the hydrophile swelling granules distributed within the medium have several important effects on the system:

(i) they capture and immobilize part of the liquid,

(ii) by increasing their volume (up to 60 times the initial value) they modify the porosity of the medium,

(iii) they become active as soon as they are reached by the wetting front (thus keeping memory of such event) and through their swelling kinetics they bring the history of the flow into the main rheological coefficients of the system.

A large discussion about modelling the various physical situations possibly occurring during this process can be found in [7]. A partial solution to the one-dimensional problem, referring to unsaturated flow, has been given in [8]. Substantial generalizations are [6, 10,11], all dealing with the one-dimensional flow.

Corresponding author. Email: andro@numerix.univ-lyon1.fr 
The present paper deals with a 3D flow through a porous material in which the hydrophile granules are represented by balls with fixed centre and time-dependent radius, whose size is not neglected on the scale of the flow.

The system is schematized as a cube of side $L$ (the porous material) with a regular array of hydrophile balls. In the Cartesian coordinates $x_{1}, x_{2}, x_{3}$ the sides $x_{1}=0, x_{1}=L$ represent the inflow and outflow boundaries, respectively. No flow takes places through the lateral boundaries. Pressure is prescribed at $x_{1}=0$. We assume that the surface $x_{1}=L$ is impervious (this is the real condition for diapers).

The balls centers occupy the positions of coordinates $\left(\frac{\epsilon}{2}, \frac{\epsilon}{2}, \frac{\epsilon}{2}\right)+(i \epsilon, j \epsilon, k \epsilon)$ with $\epsilon=$ $\frac{L}{N}, i, j, k \in \mathbf{Z}$, chosen so that all centers are inner points. We denote by $B_{i j k}(t)$ the generic ball and by $R_{i j k}(t)$ its radius, ranging from the initial value $R_{\min }$ and the maximum admissible value $R_{\max }<\epsilon / 2$, so that the spheres will always be separated by a positive distance. We also require that $R_{\max }$ is such that the medium with completely swollen spheres has a positive porosity.

The radii vary according to a kinetics driven by the surrounding values of the moisture content, that will be described in the next section. The most delicate question in the model, i.e. the definition of porosity as a function of time and space during the swelling of the spheres, will be analysed in the next section, where the mathematical problem will be formulated in its differential form.

The mathematical model is a degenerate non-linear parabolic equation in a geometry evolving with the solution. Degenerate parabolic equations, in dimensions larger than 1 , are usually studied using two different approaches: (i) $L^{1}$-contractions and (ii) compactness methods based on the topology induced by the energy integral together with estimates on the time differences. The first approach is followed in classical works by Benilan, Crandall et al.. It allows precise results for scalar problems and there was a recent breakthrough concerning uniqueness. For more details the reader should consult [15] and [4] and references therein.

The second approach was introduced by H. W. Alt and S. Luckhaus in [2]. It was used with success not only for scalar degenerate parabolics but also for degenerate coupled systems arising in important applications (see e.g. $[1,9,14]$ and references therein). In the problem which will be formulated in the next section, the geometry changes in time and it is not evident how to apply the non-linear semi-groups technique. At the same time one should take care of the equation describing evolution of the geometry. Hence it is natural to choose the tools introduced in [2].

The differential formulation is discretized using backward time differences in Section 3. Then in Section 4 we prove the existence of a solution for the discretized problem. In Sections 5 and 6 we obtain the a priori estimates. Finally, Section 7 contains passing to the limit as the time step $h$ tends to zero. To our knowledge it is the first time that a degenerate parabolic equation in a geometry evolving with the solution is considered.

\section{Evolution of the swelling spheres and of the porosity; differential formulation of the flow problem}

We denote by $p$ the pressure and by $\mathcal{S}(p)$ the saturation in the flow region. The function $\mathcal{S}$ belongs to $C^{0, \chi}(\mathbf{R})$ for some $\chi \in(0,1]$, it is monotone and moreover

$$
\begin{aligned}
& \mathcal{S}(p)=0 \quad \text { for } p<0, \\
& \mathcal{S}(p)=1 \quad \text { for } p>p_{S}>0, \\
& \mathcal{S}\left(p_{c}\right)=\mathcal{S}_{o} \in(0,1) \quad \text { for some } p_{c} \in\left(0, p_{S}\right),
\end{aligned}
$$


where $\mathcal{S}_{0}$ is supposed to be a threshold for absorption by the granules. We remark that taking $\mathcal{S}(p)=0$ for $p<0$ is not crucial. Our analysis holds true also if $\mathcal{S}(p)$ is positive everywhere. The rate of volume increase of the sphere $B_{i j k}(t)$ due to absorption is proportional to the difference $\left(\mathcal{S}-\mathcal{S}_{0}\right)_{+}$multiplied by the porosity $n(x, t)$ and integrated over its boundary.

\section{- Absorption kinetics}

$$
4 \pi R_{i j k}^{2}(t) \frac{\mathrm{d} R_{i j k}}{\mathrm{~d} t}=f\left(R_{\max }-R_{i j k}(t)\right) \int_{\partial B_{i j k}(t)} n(x, t)\left(\mathcal{S}-\mathcal{S}_{0}\right)_{+} \mathrm{d} \sigma,
$$

where $f$ is a non-negative continuously differentiable function in $\left[0, R_{\max }-R_{\min }\right]$, such that $f(0)=$ $0, f^{\prime}(z) \geqslant 0$ (and $f^{\prime}>0$ in $z=R_{\max }-R_{\min }$ ). The condition $f(0)=0$ implies that absorption stops when balls reach the maximum size.

Differently from previous models, we are keeping here the actual size of the spheres, not averaging their influence on the flow (the alternative approach would be to define $\mathcal{S}$ everywhere on the large scale, to introduce a density of the spheres and consider their average size at a macroscopic point, i.e. on a representative volume element).

The cost of not missing information at the scale of the granules is of course that kinetics (2.1) involves the saturation in a non-local way.

The physical situation for which this approach is meaningful is the one in which the balls are much larger than the microscopic elements assembled in the skeleton of the hosting porous medium (e.g. the cellulose fibres in a diaper). In this case the medium can be considered a continuum not only at the macroscopic scale, but also in the scale of $R_{\max }$.

Let us define the porosity of our evolving porous medium. We define it for each point $\left(x_{1}, x_{2}, x_{3}\right)$ that at instant $t$ does not belong to any of the spheres $B_{i j k}(t)$, i.e. in the flow domain $\Omega(t)=$ $\Omega \backslash \cup_{i, j, k} B_{i j k}(t)$, having set $\Omega=(0, L)^{3}$.

Let $n_{0}$ be the porosity of the medium in the absence of granules. The condition that for $R_{i j k}=$ $R_{\max }$ there must be some residual porosity is expressed by $\frac{4}{3} \pi R_{\max }^{3}<n_{0} \epsilon^{3}$. The corresponding minimum porosity is

$$
n_{\min }=n_{0}\left[1-\frac{4}{3} \pi\left(\frac{R_{\max }}{\epsilon}\right)^{3}\right]>0 .
$$

In order to define the porosity for general values of the radii $R_{i j k}(t)$, let us consider the cube of side $\epsilon$ with vertices at the points $\left(\frac{\epsilon}{2}, \frac{\epsilon}{2}, \frac{\epsilon}{2}\right)+\epsilon\left(i+s_{1}, j+s_{2}, k+s_{3}\right)$, with $i, j, k$ fixed and $s_{1}, s_{2}, s_{3}$ taking values 0 and 1 . The cube center has coordinates $\epsilon(i+1, j+1, k+1)$. The vertices are centers of swelling balls, each having $\frac{1}{8}$ of their volume intersecting the cube under examination. Thus a natural way of defining the porosity at the center $P_{i j k}^{o}$ is

$$
n\left(P_{i j k}^{o}\right)=n_{0}\left\{1-\frac{1}{8} \sum_{s_{1}=0}^{1} \sum_{s_{2}=0}^{1} \sum_{s_{3}=0}^{1} \frac{4}{3} \pi\left[\frac{R_{i+s_{1}, j+s_{2}, k+s_{3}}}{\epsilon}\right]^{3}\right\}
$$

\footnotetext{
For points close to the boundaries $x=0, x=L$ there may be vertices of the surrounding cube not lying in $\Omega$. In that case just give them a weight zero. Extension beyond the lateral sides can be done by periodicity.
} 
An obvious requirement for extension of the function $n$ is that it is continuous across the boundary of the cube. This implies that the size of a ball at a vertex must not influence $n$ at the points of those faces not containing that vertex. Thus we are led to introduce weights $w_{s_{1} s_{2} s_{3}}$ for each vertex depending on a suitably defined distance from that vertex of the point in which we want to define $n$.

In order to create the corresponding formula we introduce normalized Cartesian coordinates within the cube, $\xi, \eta, \zeta$, varying in $\left(-\frac{1}{2}, \frac{1}{2}\right)$, such that the center has zero coordinates and the vertices are the points $\left(-\frac{1}{2}+s_{1},-\frac{1}{2}+s_{2},-\frac{1}{2}+s_{3}\right)=\left(\xi_{s_{1}}, \eta_{s_{2}}, \zeta_{s_{3}}\right)$. For two points $P=(\xi, \eta, \zeta), P^{\prime}=$ $\left(\xi^{\prime}, \eta^{\prime}, \zeta^{\prime}\right)$ in the cube, the natural normalized distance in the system geometry is

$$
\operatorname{dist}\left(P, P^{\prime}\right)=\max \left(\left|\xi-\xi^{\prime}\right|,\left|\eta-\eta^{\prime}\right|,\left|\zeta-\zeta^{\prime}\right|\right) .
$$

Denoting by $d_{s_{1} s_{2} s_{3}}(\xi, \eta, \zeta)$ the distance of $P$ from the vertex labelled by $\left(s_{1}, s_{2}, s_{3}\right)$, we choose the weights

$$
w_{s_{1} s_{2} s_{3}}(\xi, \eta, \zeta)=\left[1-d_{s_{1} s_{2} s_{3}}(\xi, \eta, \zeta)\right]
$$

and we define the following.

\section{- Interpolated porosity}

$$
n_{i j k}(\xi, \eta, \zeta)=\left\{1-\frac{1}{Z} \sum_{s_{1}=0}^{1} \sum_{s_{2}=0}^{1} \sum_{s_{3}=0}^{1} \frac{4}{3} \pi w_{s_{1} s_{2} s_{3}}(\xi, \eta, \zeta)\left[\frac{R_{i+s_{1}, j+s_{2}, k+s_{3}}}{\epsilon}\right]^{3}\right\} n_{0} .
$$

with $Z=\sum_{s_{1}=0}^{1} \sum_{s_{2}=0}^{1} \sum_{s_{3}=0}^{1} w_{s_{1} s_{2} s_{3}}(\xi, \eta, \zeta)$.

This formula satisfies all the desired requirements. For instance at the center of the cube all weights are $\frac{1}{2}$ and $Z=4$, so that $1 / Z w_{s_{1} s_{2} s_{3}}=\frac{1}{8}$ for all vertices. At the centers of a face four weights are $\frac{1}{2}$ and four other are zero, so that $Z=2$ and the vertices of the face enter the average (2.6) with a coefficient $\frac{1}{4}$. At the center of a wedge two weights are $\frac{1}{2}$ and all the others are zero, hence $Z=1$ and the vertices on the wedge have a coefficient $\frac{1}{2}$. Therefore (2.6) is certainly a geometrically consistent definition.

By construction the function $n\left(x_{1}, x_{2}, x_{3}, t\right)$ is Lipschitz continuous in the space variables and has the same regularity as $\partial \Omega(t)$ with respect to time.

We are now ready to state the flow problem in differential form.

\section{- Flow equation}

Assuming Darcy's law for moisture displacement and neglecting gravity, the mass balance equation is

$$
\frac{\partial}{\partial t}\{n \mathcal{S}(p)\}-\operatorname{div}\{k \nabla p\}=0 \quad \text { in } \bigcup_{t \in(0, T)} \Omega(t)=Q_{T} .
$$

Here $k$ is the hydraulic permeability which is assumed to depend in a continuous fashion on the porosity $n$ and on the saturation $\mathcal{S}$

$$
k=k(n, \mathcal{S}) .
$$


More precisely, we take

$$
\left|\frac{\partial k}{\partial n}\right| \leqslant c_{1} \quad, \quad k=\widetilde{k}(\mathcal{S}) \mathcal{S}^{\beta} \quad \text { with } \quad \tilde{k} \geqslant c_{0}>0 \text { and } \beta \geqslant 0,
$$

$\widetilde{k}$ being a smooth function and $c_{0}$ a given constant.

\section{- Flow condition at the spheres boundary}

At $\partial B_{i j k}(t)$ the Darcian flux $-k \nabla p \cdot \vec{v}$ ( $\vec{v}$ unit normal vector pointing out of $\Omega(t)$ ) equals the mass transport rate due to the motion of the surface, i.e. $-n_{i j k} \mathcal{S}(p) \frac{\mathrm{d} R_{i j k}}{\mathrm{~d} t}$, plus the absorption rate $f\left(R_{\max }-R_{i j k}\right) n\left(\mathcal{S}-\mathcal{S}_{0}\right)_{+}$. Hence

$$
\left.\left(-k \nabla p \cdot \vec{v}+n \mathcal{S} \frac{\mathrm{d} R_{i j k}}{\mathrm{~d} t}\right)\right|_{\partial B_{i j k}(t)}=\left.f\left(R_{\max }-R_{i j k}\right) n\left(\mathcal{S}-\mathcal{S}_{0}\right)_{+}\right|_{\partial B_{i j k}(t)},
$$

$\forall i, j, k$.

Note that (2.10) is basically a non-local condition, in view of (2.1).

\section{- Flow conditions on the external boundaries}

As we have already said, we take

$$
\begin{aligned}
& p=P\left(x_{2}, x_{3}, t\right) \geqslant 0 \quad \text { on } x_{1}=0 \\
& k \nabla p \cdot \vec{v}=0 \quad \text { on } x_{1}=L, t>0 \\
& k \nabla p \cdot \vec{v}=0 \quad \text { on the lateral sides of the cube. }
\end{aligned}
$$

\section{- Initial conditions}

For $t=0$ we suppose that all the granules have the minimum size

$$
R_{i j k}(0)=R_{\min }
$$

and that

$$
\left.\mathcal{S}\right|_{t=0}=\mathcal{S}^{o}(x) \geqslant 0 \quad \text { on } \Omega(0) .
$$

Of course (2.14) could be easily generalized. The initial porosity is

$$
n(x, 0)=n_{\max }=n_{0}\left[1-\frac{4}{3} \pi\left(\frac{R_{\min }}{\epsilon}\right)^{3}\right]
$$

Thus the differential formulation of the problem consists of the (degenerate) parabolic equation (2.7) with the coefficients $n, k$ defined by (2.6), (2.8), of the (non-local) kinetic equation (2.1), and of the initial and boundary conditions (2.10)-(2.15). The unknowns are the pressure $p(x, t)$ and the radii $R_{i j k}$. 


\section{Weak formulation}

In order to homogenize the boundary conditions for the pressure we introduce the auxiliary function

$$
\tilde{\pi}=\left(1-\frac{x_{1}}{L}\right) P\left(x_{2}, x_{3}, t\right) .
$$

We define

$$
u(p)=\int_{0}^{p}(\mathcal{S}(\xi))^{\beta} \mathrm{d} \xi, \quad p \geqslant 0
$$

and $p(u)$ as the inverse function of $u(p)$ for $u>0$ and $p(u)=0$ for $u \leqslant 0$.

So we can also define

$$
S(u)=\mathcal{S}(p(u))
$$

We remark that $\nabla u=(S(u))^{\beta} \nabla p$ and we also note that from the initial condition (2.15) we are able to deduce the corresponding function $u^{0}(x)$ in the set $\left\{\mathcal{S}^{o}>0\right\} \subset \Omega(0)$ and we complete its definition putting $u^{o}=0$ in the complementary subset of $\Omega(0)$.

In the sequel we shall require that

(H1) $u^{o} \in L^{q}(\Omega(0))$, for some $q>1$

(H2) $P \in H^{1}\left(\left\{x_{1}=0\right\} \times(0, T)\right), \quad P \geqslant 0$ a.e.

Now we state the weak formulation of the problem for $u$.

Problem $(P) \quad$ Find $u \in L^{2}\left(0, T ; H^{1}(\Omega)\right), u \geqslant 0$ a.e. on $\Omega(t) \times(0, T)$ and $R_{i j k} \in C^{0,1}([0, T])$ satisfying

$$
\begin{aligned}
& \int_{0}^{T} \int_{\Omega(t)}\left(\tilde{k} \nabla u \nabla \varphi-n S(u) \frac{\partial \varphi}{\partial t}\right) \mathrm{d} x \mathrm{~d} t \\
& \left.+\sum_{i j k} \int_{0}^{T} f\left(R_{\max }-R_{i j k}\right)(t)\right) \int_{\partial B_{i j k}(t)} \varphi n\left(S(u)-S_{0}\right)_{+} \mathrm{d} \sigma \mathrm{d} t \\
& -\int_{\Omega(0)} n_{\max } \mathcal{S}^{o} \varphi(x, 0) \mathrm{d} x=0
\end{aligned}
$$

for all test functions $\varphi$ in the class

$$
\varphi \in H^{1}(\Omega \times(0, T)), \quad \varphi(x, T)=0 \text { and }\left.\varphi\right|_{x_{1}=0}=0 .
$$

Moreover, the equations (2.1), (2.14) hold true for $R_{i j k}, n$ is given by (2.6) and

$$
\begin{aligned}
& \int_{0}^{T}\left\langle\frac{\partial}{\partial t}(n S), \varphi\right\rangle \mathrm{d} t=-\int_{0}^{T} \int_{\Omega(t)} n S \frac{\partial \varphi}{\partial t} \mathrm{~d} x \mathrm{~d} t \\
& -\int_{\Omega(0)} n_{\max } \mathcal{S}^{o} \varphi(x, 0) \mathrm{d} x+\int_{0}^{T} \sum_{i, j, k} \frac{\mathrm{d} R_{i j k}}{\mathrm{~d} t} \int_{\partial B_{i j k}(t)} \varphi n S(u) \mathrm{d} \sigma \mathrm{d} t
\end{aligned}
$$

for all $\varphi$ in the class (3.5).

For a given classical solution it is easy to derive (3.4), multiplying by $\varphi$ the flow equation, integrating and using (2.1) and the initial and boundary conditions. 
REMARK 1 Due to particular way of the evolution of the balls all functions are considered to be defined on $\Omega$. More precisely, since the radii of the balls grow between $R_{\min }$ and $R_{\max }$ and they never touch each other, it is possible to use the Extension lemma (see e.g. [13], page 88) and extend a function $g \in H^{1}(\Omega(t))$ to a function $\tilde{g} \in H^{1}(\Omega)$, coinciding with $g$ in $\Omega(t)$ a.e. and such that

$$
\int_{\Omega}|\nabla \tilde{g}|^{2} \mathrm{~d} x \leqslant c_{0} \int_{\Omega(t)}|\nabla g|^{2} \mathrm{~d} x
$$

and

$$
\int_{\Omega}|\tilde{g}|^{2} \mathrm{~d} x \leqslant c_{0} \int_{\Omega(t)}|g|^{2} \mathrm{~d} x,
$$

where $c_{0}$ doesn't depend on $t$. We note that the Extension lemma is frequently used in homogenization problems. To the author's knowledge, it was first proved in [5] using an idea of L. Tartar.

\section{The discretized problem}

We discretize in time with a sufficiently small time step $h>0$ and the backward difference quotient is denoted $\partial_{t}^{-h}$.

Let $V=\left\{z \in H^{1}(\Omega) \mid z=0\right.$ for $\left.x_{1}=0\right\}$. We define $\Omega^{l}=\Omega(l h)$ and choose a $C^{1}(\bar{\Omega})$-basis $\left\{\varphi_{m}\right\}$ for $V$. We note that $l$ labels the time step.

We are looking for a finite-dimensional approximate solution for the problem $(\mathrm{P})$ in the form

$$
u_{h N}(x, t)=\sum_{m=1}^{N} \alpha_{h N m}(t) \varphi_{n}(x)+u(\tilde{\pi}),
$$

i.e. for $\alpha_{h N m}$ piece wise constant in $t$, solving

$$
\begin{aligned}
& \int_{\Omega^{j}}\left[n^{j} \partial_{t}^{-h} S\left(u^{j}\right)+\partial_{t}^{-h} n^{j} S\left(u^{j-1}\right)\right] \zeta \mathrm{d} x \\
& +\int_{\Omega^{j-1}} \widetilde{k}^{j} \nabla u^{j} \nabla \zeta \mathrm{d} x+\sum_{i m k} \int_{\partial B_{i m k}^{j}} \zeta n^{j} f\left(R_{\max }-R_{i m k}^{j}\right) . \\
& \times\left\{\left(S\left(u^{j}\right)-S_{0}\right)_{+}-S\left(u^{j}\right) \int_{\partial B_{i m k}^{j}} n^{j}\left(S\left(u^{j}\right)-S_{0}\right)_{+}\right\} \mathrm{d} \sigma=0
\end{aligned}
$$

$\forall \zeta \in V_{N}$, where $\int$ denotes the average, $V_{N}=\operatorname{span}\left\{\varphi_{1}, \ldots, \varphi_{N}\right\}$ and $B_{i m k}^{j}$ is the ball $B_{i m k}(j h)$.

By definition

$$
\begin{aligned}
& \Omega^{j}=\Omega^{j-1} \backslash \bigcup_{i m k} B_{i m k}^{j}, \quad j=1, \ldots, k \\
& u^{j}=u_{h N}(x, j h), \quad j=1, \ldots, T / h,
\end{aligned}
$$

$\tilde{k}^{j}=\tilde{k}\left(S\left(u^{j}\right)\right)$ and $n^{j}$ is given by (2.6), with $R^{j}$ replacing $R$. 
The discretized evolution law for $R_{i m k}^{j}=R_{i m k}(\tau)$ on $((j-1) h, j h]$ is

$$
\begin{aligned}
4 \pi\left(R_{i m k}^{j}\right)^{2} R_{i m k}^{j}= & 4 \pi\left(R_{i m k}^{j-1}\right)^{3} \\
& +h f\left(R_{\max }-R_{i m k}^{j-1}\right) \int_{\partial B_{i m k}^{j}} n^{j}\left(S\left(u^{j}\right)-S_{0}\right)_{+} \mathrm{d} \sigma,
\end{aligned}
$$

where we have dropped the index $N$ to simplify notation.

The initial conditions specifying $\mathcal{S}^{o}$ and $R_{i m k}^{o}$ are the same as for the original variables, i.e. (2.14), (2.15).

Our first step is to show existence for the discretized problem. We will make use of the following auxiliary result.

LEMMA 1 Let $\left\{C_{i}\right\}$ be a periodic array of balls, which do not touch each other, over a cubic lattice. Let $\widetilde{B}_{i}$ be the union of the cells containing $C_{i}$ and its neighbours. Then for all $\varphi \in B V\left(\tilde{B}_{i}\right)$

$$
\int_{\partial C_{i}}|\varphi| \mathrm{d} \sigma \leqslant \bar{C}\left\{\int_{\widetilde{B}_{i} \backslash C_{i}}|\nabla \varphi| \mathrm{d} y+\int_{\widetilde{B}_{i} \backslash C_{i}}|\varphi| \mathrm{d} y\right\} .
$$

For a proof see [12].

Now we prove the following result

THEOREM 1 Suppose $u^{j}$ is known for all $j<k$. Then the system (4.1)-(4.5), together with the initial condition, has at least one solution $u^{k}$.

REMARK 2 As usual we are going to use a variant of Brouwer's fixed-point theorem for proving Theorem 1. The energy has a correct growth and at first glance everything looks easy. Nevertheless, any change in the solution implies a change of the geometry and the main difficulty is to prove that it doesn't alter the coerciveness of the energy. Therefore we discuss the whole procedure at some length.

Proof. For all $\mu \in \mathbf{R}^{N}$ we define

$$
v_{\mu}(x)=\sum_{m=1}^{N} \mu_{m} \varphi_{m}(x)+u(\tilde{\pi}), \quad \operatorname{meas}\left(\operatorname{supp} \varphi_{m} \cap \Omega^{k}\right)>0
$$

where $\tilde{\pi}$ is given by (3.1). Then we study the following system of non-linear algebraic equations for $\mu$ :

$$
\begin{aligned}
& \Phi_{m}(\mu)=\int_{\Omega^{\mu}} n^{\mu}\left\{S\left(v_{\mu}\right)-S\left(u^{k-1}\right)\right\} \varphi_{m} \mathrm{~d} x \\
& +h \int_{\Omega^{k-1}} \widetilde{k}^{\mu} \nabla v_{\mu} \nabla \varphi_{m} \mathrm{~d} x+h \int_{\Omega^{\mu}} \partial_{t}^{-h} n^{\mu} S\left(u^{k-1}\right) \varphi_{m} \mathrm{~d} x \\
& +h \sum_{i, j, l} \int_{\partial B_{i, j, l}^{\mu}} \varphi_{m} n^{\mu} f\left(R_{\max }-R_{i j l}^{\mu}\right)\left\{\left(S\left(v_{\mu}\right)-S_{0}\right)_{+}\right. \\
& \left.-S\left(v_{\mu}\right) \int_{\partial B_{i j l}^{\mu}} n^{\mu}\left(S\left(v_{\mu}\right)-S_{0}\right)_{+}\right\} \mathrm{d} \sigma=0 .
\end{aligned}
$$


It should be noted that $\Omega^{\mu}$ is the flow region corresponding to the balls with radii $R_{i m k}^{\mu}$, given by (4.5) with $u^{j}=v_{\mu}$ and $R_{i m k}^{j-1}=R_{i m k}^{k-1}$. Now we compute $\Phi(\mu) \cdot \mu$ :

$$
\begin{aligned}
& \Phi(\mu) \cdot \mu=\int_{\Omega^{k}} n^{\mu}\left\{S\left(v_{\mu}\right)-S\left(u^{k-1}\right)\right\}\left(v_{\mu}-u(\tilde{\pi})\right) \mathrm{d} x \\
& +h \int_{\Omega^{k-1}} \widetilde{k}^{\mu} \nabla v_{\mu} \nabla\left(v_{\mu}-u(\tilde{\pi})\right) \mathrm{d} x+h \int_{\Omega^{\mu}} \partial_{t}^{-h} n^{\mu} S\left(u^{k-1}\right)\left(v_{\mu}-u(\tilde{\pi})\right) \mathrm{d} x \\
& +h \sum_{i j l} \int_{\partial B_{i j l}^{\mu}} n^{\mu}\left(v_{\mu}-u(\tilde{\pi})\right) f\left(R_{\max }-R_{i j l}^{\mu}\right)\left\{\left(S\left(v_{\mu}-S_{0}\right)_{+}\right.\right. \\
& \left.-S\left(v_{\mu}\right) \int_{\partial B_{i j l}^{\mu}} n^{\mu}\left(S\left(v_{\mu}\right)-S_{0}\right)_{+}\right\} \mathrm{d} \sigma .
\end{aligned}
$$

We note that showing the existence of $u^{k}$ is equivalent to proving that $\Phi(\mu)$ has at least one zero. This is guaranteed if $\Phi(\mu) \cdot \mu$ grows quadratically as $|\mu| \rightarrow+\infty$.

With this aim in mind we first note that $0 \leqslant f\left(R_{\max }-R_{i j l}^{\mu}\right) \leqslant \epsilon C$ implies

$$
\begin{aligned}
& I_{1}=h \mid \sum_{i j l} \int_{\partial B_{i j l}^{\mu}} v_{\mu} n^{\mu} f\left(R_{\max }-R_{i j l}^{\mu}\right)\left\{\left(S\left(v_{\mu}\right)-S_{0}\right)_{+}\right. \\
& \left.-S\left(v_{\mu}\right) \int_{\partial B_{i j l}^{\mu}} n^{\mu}\left(S\left(v_{\mu}\right)-S_{0}\right)_{+}\right\} d \sigma\left|\leqslant C_{1} \epsilon h \sum_{i j l} \int_{\partial B_{i j l}^{\mu}}\right| v_{\mu} \mid \mathrm{d} \sigma
\end{aligned}
$$

and we use Lemma 1 to obtain

$$
I_{1} \leqslant C_{2} h \int_{\Omega^{\mu}}\left(\epsilon\left|\nabla v_{\mu}\right|+\left|v_{\mu}\right|\right) \mathrm{d} x .
$$

Having estimated the surface integral in (4.9), we turn our attention to the term

$$
h \int_{\Omega^{\mu}} \partial_{t}^{-h} n^{\mu} S\left(u^{k-1}\right)\left(v_{\mu}-u(\tilde{\pi})\right) \mathrm{d} x
$$

and we observe that, owing to (2.6), $\partial_{t}^{-h} n^{\mu}$ is a weighted average of $\partial_{t}^{-h}\left(\frac{R_{i j l}^{\mu}}{\epsilon}\right)^{3}$ at the corners of a cell. In view of (4.5) we have $\left|\partial_{t}^{-h} n^{\mu}\right| \leqslant C_{3}$ and, consequently,

$$
\begin{aligned}
& I_{2}=\left|h \int_{\Omega^{\mu}} \partial_{t}^{-h} n^{\mu} S\left(u^{k-1}\right)\left(v_{\mu}-u(\tilde{\pi})\right) \mathrm{d} x\right| \\
& \leqslant h C_{4} \int_{\Omega^{\mu}}\left|v_{\mu}\right| \mathrm{d} x+h C_{5} .
\end{aligned}
$$

Since $\int_{\Omega^{\mu}} n^{\mu}\left\{S\left(v_{\mu}\right)-S\left(u^{k-1}\right)\right\}\left(v_{\mu}-u(\tilde{\pi})\right) \mathrm{d} x$ is bounded from below by a constant $-C_{6}$, we set

$$
\begin{aligned}
\Phi(\mu) \cdot \mu \geqslant & h \int_{\Omega^{k-1}} \widetilde{k}^{\mu}\left|\nabla v_{\mu}\right|^{2} \mathrm{~d} x-C_{2} h \int_{\Omega^{\mu}}\left(\epsilon\left|\nabla v_{\mu}\right|+\left|v_{\mu}\right|\right) \mathrm{d} x \\
& -h C_{4} \int_{\Omega^{\mu}}\left|v_{\mu}\right| \mathrm{d} x-C_{7} .
\end{aligned}
$$

Now Poincaré's inequality implies that $\Phi(\mu) \cdot \mu$ behaves as $C_{0}|\mu|^{2}$, for large $|\mu|$ and the theorem is proved. 


\section{A priori estimate in the space variable}

Following [2] we define

$$
B(z)=z S(z)-\int_{0}^{z} S(\xi) \mathrm{d} \xi \geqslant 0
$$

and we prove the following estimate.

LEMMA 2 For all $N$ we have

$$
\sup _{0 \leqslant t \leqslant T} \int_{\Omega^{N}(t)} n B\left(u_{h N}\right) \mathrm{d} x+\int_{0}^{T} \int_{\Omega^{N}(t)} \tilde{k}\left|\nabla u_{h N}\right|^{2} \mathrm{~d} x \mathrm{~d} t \leqslant C
$$

for some constant $C>0$, independent of $N$.

REMARK 3 The estimate (5.2) corresponds to the energy estimate from [2]. Nevertheless, for an evolving structure, the discretized integral of the function $n B$ is more complicated and composed of the integrals over $\Omega^{j} \backslash \Omega^{j+1}$. In contrast with [2], we have to control the changing geometry and the monotone growth of the balls is used in an essential way.

Proof. Take $\zeta=u^{j}-u(\tilde{\pi})$ in (4.2) and sum from 1 to $j$ :

$$
\begin{aligned}
& \sum_{j=1}^{k} \int_{\Omega^{j}} \frac{1}{h}\left\{n^{j}\left[S\left(u^{j}\right)-S\left(u^{j-1}\right)\right]+\left(n^{j}-n^{j-1}\right) S\left(u^{j-1}\right)\right\}\left(u^{j}-u(\tilde{\pi})\right) \mathrm{d} x \\
& +\sum_{j=1}^{k} \int_{\Omega^{j-1}} \tilde{k}^{j} \nabla u^{j} \cdot \nabla\left(u^{j}-u(\tilde{\pi})\right) \mathrm{d} x \\
& +\sum_{j=1}^{k} \sum_{i m l} \int_{\partial B_{i m l}^{j}} n^{j}\left(u^{j}-u(\tilde{\pi})\right) f\left(R_{\max }-R_{i m l}^{j}\right) . \\
& \times\left\{\left[S\left(u^{j}\right)-S_{0}\right]_{+}-S\left(u^{j}\right) \oint_{\partial B_{i m l}^{j}} n^{j}\left[S\left(u^{j}\right)-S_{0}\right]_{+}\right\} \mathrm{d} \sigma=0
\end{aligned}
$$

where $S\left(u^{0}\right)=\mathcal{S}^{0}$ from $(2.15)$

We put the terms involving $u(\tilde{\pi})$ on the right-hand side and start estimating the left-hand side. We denote the right-hand side by $J$. 
The first term can be rewritten as

$$
\begin{aligned}
& \frac{1}{h} \sum_{j=1}^{k-1}\left\{\int_{\Omega^{j}} n^{j} S\left(u^{j}\right) u^{j} \mathrm{~d} x-\int_{\Omega^{j+1}} n^{j+1} S\left(u^{j}\right) u^{j+1} \mathrm{~d} x\right\} \\
& +\frac{1}{h} \int_{\Omega^{k}} n^{k} S\left(u^{k}\right) u^{k} \mathrm{~d} x-\frac{1}{h} \int_{\Omega(h)} n^{1} S\left(u^{0}\right) u^{1} \mathrm{~d} x \\
= & \sum_{j=1}^{k} \int_{\Omega^{j+1}} n^{j} S\left(u^{j}\right) \frac{1}{h}\left(u^{j}-u^{j+1}\right) \mathrm{d} x+\frac{1}{h} \int_{\Omega^{k}} n^{k} S\left(u^{k}\right) u^{k} \mathrm{~d} x \\
& -\frac{1}{h} \int_{\Omega^{1}} n^{1} S\left(u^{0}\right) u^{1} \mathrm{~d} x+\frac{1}{h} \sum_{j=1}^{k-1} \int_{\Omega^{j} \backslash \Omega^{j+1}} n^{j} S\left(u^{j}\right) u^{j} \mathrm{~d} x \\
& -\sum_{j=1}^{k-1} \int_{\Omega^{j+1}} \partial_{t}^{-h} n^{j+1} S\left(u^{j}\right) u^{j+1} \mathrm{~d} x .
\end{aligned}
$$

We note that

$$
S\left(u^{j}\right)\left(u^{j}-u^{j+1}\right) \geqslant-\int_{u^{j}}^{u^{j+1}} S(\xi) \mathrm{d} \xi
$$

because $S$ is increasing. Therefore from (5.3)-(5.5) we obtain the inequality

$$
\begin{aligned}
h J \geqslant & -\sum_{j=1}^{k-1} \int_{\Omega^{j+1}} n^{j} \int_{u^{j}}^{u^{j+1}} S(\xi) \mathrm{d} \xi \mathrm{d} x+\int_{\Omega^{k}} n^{k} S\left(u^{k}\right) u^{k} \mathrm{~d} x \\
& -\int_{\Omega^{1}} n^{1} S\left(u^{0}\right) u^{1} \mathrm{~d} x+\sum_{j=1}^{k-1} \int_{\Omega^{j} \backslash \Omega^{j+1}} n^{j} S\left(u^{j}\right) u^{j} \mathrm{~d} x \\
& +h \sum_{j=1}^{k} \int_{\Omega^{j-1}} \tilde{k}^{j}\left|\nabla u^{j}\right|^{2} \mathrm{~d} x+h \int_{\Omega^{1}} \partial_{t}^{-h} n^{1} S\left(u^{0}\right) u^{1} \mathrm{~d} x \\
& +h \sum_{j=1}^{k} \sum_{i m l} \int_{\partial B_{i m l}^{j}} n^{j} u^{j} f\left(R_{\max }-R_{i m l}^{j}\right)\left\{\left(S\left(u^{j}\right)-S_{0}\right)_{+}\right. \\
& \left.-S\left(u^{j}\right) \int_{\partial B_{i m l}^{j}} n^{j}\left(S\left(u^{j}\right)-S_{0}\right)_{+}\right\} \mathrm{d} \sigma .
\end{aligned}
$$

Observing that

$$
\int_{\Omega^{j} \backslash \Omega^{j+1}}=\int_{\Omega^{j}}-\int_{\Omega^{j+1}}
$$


by re-labeling the first terms we obtain

$$
\begin{aligned}
& \sum_{j=1}^{k-1} \int_{\Omega^{j} \backslash \Omega^{j+1}} n^{j} S\left(u^{j}\right) u^{j} \mathrm{~d} x \\
= & \sum_{j=2}^{k-2} \int_{\Omega^{j+1}}\left[n^{j+1} S\left(u^{j+1}\right) u^{j+1}-n^{j} S\left(u^{j}\right) u^{j}\right] \mathrm{d} x \\
& -\int_{\Omega^{k}} n^{k-1} S\left(u^{k-1}\right) u^{k-1} \mathrm{~d} x+\int_{\Omega^{1}} n^{1} S\left(u^{1}\right) u^{1} \mathrm{~d} x \\
= & \sum_{j=1}^{k-1} \int_{\Omega^{j+1}}\left(n^{j+1}-n^{j}\right) S\left(u^{j+1}\right) u^{j+1} \mathrm{~d} x \\
& +\sum_{j=1}^{k-1} \int_{\Omega^{j+1}} n^{j}\left[S\left(u^{j+1}\right) u^{j+1}-S\left(u^{j}\right) u^{j}\right] \mathrm{d} x \\
& +\int_{\Omega^{1}}\left[n^{1} S\left(u^{1}\right)-n^{0} S\left(u^{0}\right)\right] u^{1} \mathrm{~d} x-\int_{\Omega^{k}} n^{k} S\left(u^{k}\right) u^{k} \mathrm{~d} x+\int_{\Omega^{1}} n^{0} S\left(u^{0}\right) u^{1} \mathrm{~d} x .
\end{aligned}
$$

Substituting (5.7) in (5.6) gives

$$
\begin{aligned}
h J & \geqslant \sum_{j=1}^{k} \int_{\Omega^{j+1}} n^{j}\left\{S\left(u^{j+1}\right) u^{j+1}-S\left(u^{j}\right) u^{j}-\int_{u^{j}}^{u^{j+1}} S(\xi) \mathrm{d} \xi\right\} \mathrm{d} x \\
& +\sum_{j=1}^{k-1} \int_{\Omega^{j+1}}\left(n^{j+1}-n^{j}\right) S\left(u^{j+1}\right) u^{j+1} \mathrm{~d} x+\int_{\Omega^{1}}\left[n^{1} S\left(u^{1}\right)-n^{0} S\left(u^{0}\right) u^{1}\right] \mathrm{d} x \\
& +\int_{\Omega^{1}}\left(n^{1}-n^{0}\right) S\left(u^{0}\right) u^{1} \mathrm{~d} x+h \sum_{j=1}^{k} \int_{\Omega^{j-1}} \tilde{k}^{j}\left|\nabla u^{j}\right|^{2} \mathrm{~d} x \\
& +h \sum_{j=1}^{k} \sum_{i m l} \int_{\partial B_{i m l}^{j}} n^{j} u^{j} f\left(R_{\max }-R_{i m l}^{j}\right)\left\{\left(S\left(u^{j}\right)-S_{0}\right)_{+}\right. \\
& \left.-S\left(u^{j}\right) \int_{\partial B_{i m l}^{j}} n^{j}\left[S\left(u^{j}\right)-S_{0}\right]_{+}\right\} \mathrm{d} \sigma .
\end{aligned}
$$

We note that the first term in $(5.8)$ is

$$
\sum_{j=1}^{k-1} \int_{\Omega^{j+1}} n^{j}\left\{B\left(u^{j+1}\right)-B\left(u^{j}\right)\right\} \mathrm{d} x
$$

(remember (5.1)), which by means of the usual re-labeling technique can be given the form

$$
\begin{aligned}
& \sum_{j=2}^{k-1} \int_{\Omega^{j} \backslash \Omega^{j+1}} n^{j} B\left(u^{j}\right) \mathrm{d} x+\int_{\Omega^{k}} n^{k-1} B\left(u^{k}\right) \mathrm{d} x-\int_{\Omega^{2}} n^{1} B\left(u^{1}\right) \mathrm{d} x \\
& +\sum_{j=2}^{k-1} \int_{\Omega^{j}}\left(n^{j-1}-n^{j}\right) B\left(u^{j}\right) \mathrm{d} x,
\end{aligned}
$$


where the first and the last terms are positive.

It remains to estimate the last term in (5.8). The absolute value of each of the integrals is less than $C \epsilon \int_{\partial B_{i m l}^{j}}\left|u^{j}\right| \mathrm{d} \sigma$, to which we may apply Lemma 1 , so that

$$
\begin{aligned}
& \quad h \sum_{j=1}^{k} \sum_{i m l} \mid \int_{\partial B_{i m l}^{j}} n^{j} u^{j} f\left(R_{\max }-R_{i m l}^{j}\right)\left\{\left(S\left(u^{j}\right)-S_{0}\right)_{+}\right. \\
& \left.-S\left(u^{j}\right) \int_{\partial B_{i m l}^{j}} n^{j}\left(S\left(u^{j}\right)-S_{0}\right)_{+}\right\} \mathrm{d} \sigma \mid \leqslant \\
& \leqslant C h \sum_{j=1}^{k} \int_{\Omega^{j}}\left(\epsilon\left|\nabla u^{j}\right|+\left|u^{j}\right|\right) \mathrm{d} x .
\end{aligned}
$$

Concerning the terms involving $u(\tilde{\pi})$ in (5.3), we note that

$$
\begin{aligned}
& \mid h \sum_{j=1}^{k} \sum_{i m l} \int_{\partial B_{i m l}^{j}} n^{j} u(\tilde{\pi}) f\left(R_{\max }-R_{i m l}^{j}\right)\left\{\left(S\left(u^{j}\right)-S_{0}\right)_{+}\right. \\
& \left.-S\left(u^{j}\right) \int_{\partial B_{i m l}^{j}}\left(S\left(u^{j}\right)-S_{0}\right)_{+}\right\} \mathrm{d} \sigma \mid \leqslant C
\end{aligned}
$$

and

$$
h \sum_{j=1}^{k}\left|\int_{\Omega^{j-1}} \tilde{k}^{j} \nabla u^{j} \cdot \nabla u(\tilde{\pi}) \mathrm{d} x\right| \leqslant \delta \int_{0}^{T} \int_{\Omega^{j-1}} \tilde{k}^{j}\left|\nabla u^{j}\right|^{2} \mathrm{~d} x+\frac{C}{\delta}
$$

for some $\delta>0$ sufficiently small. Furthermore,

$$
\begin{aligned}
& h\left|\sum_{j=1}^{k} \int_{\Omega^{j}} \partial_{t}^{-h} n^{j} S\left(u^{j-1}\right) u(\tilde{\pi}) \mathrm{d} x\right| \leqslant C, \\
& \left|\sum_{j=1}^{k}\right| \int_{\Omega^{j}} n^{j}\left(S\left(u^{j}\right)-S\left(u^{j-1}\right)\right) u(\tilde{\pi}) \mathrm{d} x \mid \\
= & \mid \sum_{j=1}^{k-1} h \int_{\Omega^{j+1}} \frac{n^{j}-n^{j+1}}{h} S\left(u^{j}\right) u(\tilde{\pi}) \mathrm{d} x+\int_{\Omega^{k}} n^{k} S\left(u^{k}\right) u(\tilde{\pi}) \mathrm{d} x \\
& -\int_{\Omega^{1}} n^{1} \mathcal{S}^{0} u(\tilde{\pi}) \mathrm{d} x+\sum_{j=0}^{k-1} \int_{\Omega^{j} \backslash \Omega^{j+1}} n^{j+1} S\left(u^{j}\right) u(\tilde{\pi}) \mathrm{d} x \mid \leqslant C .
\end{aligned}
$$

At this point it is not difficult to derive the desired estimate (5.2) from (5.8).

Problem (4.1)-(4.5) could be viewed as the discretized weak form of an elliptic system. It has a solution $\left\{u_{N}^{j}\right\}_{j=1}^{T / h}$, where, according to (4.4), $u_{N}^{j}(x)=u_{h N}(x, j h)$, satisfying the a priori estimate (5.2). Elementary compactness implies the existence of a subsequence, denoted again $\left\{u_{N}^{j}\right\}_{j=1}^{T / h}$ and the limit $\left\{u^{j}\right\}_{j=1}^{T / h}$ such that

$$
u_{N}^{j} \rightarrow u^{j} \quad \text { weakly in } H^{1}(\Omega) \text {, strongly in } L^{2}(\Omega) \text {, a.e. on } \Omega \text { and } \partial B_{i m l}^{j} .
$$


It is obvious that $\left\{u^{j}\right\}_{j=1}^{T / h}$ satisfies the variational equation

$$
\begin{aligned}
& \int_{\Omega^{j}}\left[n^{j} \partial_{t}^{-h} S\left(u^{j}\right)+\partial_{t}^{-h} n^{j} S\left(u^{j-1}\right)\right] \zeta \mathrm{d} x \\
& +\int_{\Omega^{j-1}} \tilde{k}^{j} \nabla u^{j} \nabla \zeta+\sum_{i m k} \int_{\partial B_{i m k}^{j}} \zeta n^{j} f\left(R_{\max }-R_{i m k}^{j}\right) \\
& \left\{\left(S\left(u^{j}\right)-S_{0}\right)_{+}-S\left(u^{j}\right) \int_{\partial B_{i m k}^{j}} n^{j}\left(S\left(u^{j}\right)-S_{0}\right)_{+}\right\} \mathrm{d} \sigma=0
\end{aligned}
$$

$\forall \zeta \in V$ and

$$
4 \pi\left(R_{i m k}^{j}\right)^{3}=4 \pi\left(R_{i m k}^{j-1}\right)^{3}+h f\left(R_{\max }-R_{i m k}^{j-1}\right) \int_{\partial B_{i m k}^{j}} n^{j}\left[S\left(u^{j}\right)-S_{0}\right]_{+} \mathrm{d} \sigma
$$

and the same initial conditions.

Then we have the following result about the sign of $u$.

LEMMA $3 u^{j} \geqslant 0$ a.e. on $\Omega$ and on $\partial B_{i m l}^{j}$ for every $j \in\{1, \ldots, T / h\}$.

Proof. We take $\zeta=\inf \left\{u^{j}, 0\right\} \in V$ as a test function in (5.9). Since

$$
\inf \left\{u^{j}, 0\right\} \cdot S\left(u^{j}\right)=0 \quad \text { a.e. in } \Omega \text { and on } \partial B_{i m l}^{j},
$$

$\partial_{t}^{-h} n^{j} \leqslant 0$ and $-S\left(u^{j-1}\right) \inf \left\{u^{j}, 0\right\} \geqslant 0$, the equation (5.9) transforms into

$$
\int_{\Omega^{j-1}} \tilde{k}^{j}\left|\nabla \inf \left\{u^{j}, 0\right\}\right|^{2} \mathrm{~d} x \leqslant 0
$$

Consequently, $u^{j} \geqslant 0$ a.e.

\section{A priori estimate in time}

In this section we obtain an estimate for the time differences. It is essential for getting the compactness. We follow the approach from [2], but presence of an evolving geometry forces us to work out a number of additional estimates.

Let $k \in \mathbf{N}, \tau \in(0, T), \tau+k h \leqslant T$, and $\tau_{j}=\tau+j h, j=1, \ldots, k$. For simplicity we denote $S\left(u^{j}\right)$ as $S^{j}$. Then the following estimate holds.

Proposition 1 For every $\eta_{0} \geqslant \eta>0$ we have

$$
\int_{0}^{T-\eta} \int_{\Omega(\tau+\eta)}\left|S\left(u_{h}(\tau+\eta)\right)-S\left(u_{h}(\tau)\right)\right| \mathrm{d} x \mathrm{~d} \tau \leqslant C \eta^{\frac{5 \chi}{6(1+\chi(1+\beta))}}
$$

where $u_{h}(\tau)=u^{j}(x)$ for $(j-1) h<\tau \leqslant j h$. 
Proof. Let $\zeta$ be independent of $j$. Then we have

$$
\begin{aligned}
0= & \sum_{j=1}^{k} \int_{\Omega^{j}} n^{j}\left(S^{j}-S^{j+1}\right) \zeta \mathrm{d} x+h \sum_{j=1}^{k} \int_{\Omega^{j-1}} \tilde{k}^{j} \nabla u^{j} \nabla \zeta \mathrm{d} x \\
& +\sum_{j=1}^{k} \int_{\Omega^{j}}\left(n^{j}-n^{j-1}\right) S^{j-1} \zeta \mathrm{d} x \\
& +h \sum_{j=1}^{k} \sum_{i m l} \int_{\partial B_{i m l}^{j}} \zeta n^{j} f\left(R_{\max }-R_{i m l}^{j}\right) \\
& \times\left\{\left(S\left(u^{j}\right)-S_{0}\right)_{+}-S\left(u^{j}\right) \int_{\partial B_{i m l}^{j}} n^{j}\left(S\left(u^{j}\right)-S_{0}\right)_{+}\right\} \mathrm{d} \sigma
\end{aligned}
$$

leading to

$$
\begin{aligned}
0= & h \sum_{j=1}^{k} \int_{\Omega^{j-1}} \widetilde{k}^{j} \nabla u^{j} \nabla \zeta \mathrm{d} x+\int_{\Omega^{k}} n^{k} S^{k} \zeta \mathrm{d} x \\
& +\sum_{j=2}^{k} \int_{\Omega^{j-1} \backslash \Omega^{j}} S^{j-1} \zeta n^{j-1} \mathrm{~d} x \\
& -\int_{\Omega^{k}} n(\tau) S^{0} \zeta \mathrm{d} x+\sum_{j=1}^{k} \sum_{i m l} \int_{\partial B_{i m l}^{j}} \zeta n^{j} f\left(R_{\max }-R_{i m l}^{j}\right) \\
& \times\left\{\left(S\left(u^{j}\right)-S_{0}\right)_{+}-S\left(u^{j}\right) \int_{\partial B_{i m l}^{j}} n^{j}\left(S\left(u^{j}\right)-S_{0}\right)_{+}\right\} \mathrm{d} \sigma .
\end{aligned}
$$

Now we take $\zeta=u^{k}-u^{0}+\Pi^{k}-\Pi^{0}$, where $\Pi^{k}=u(\tilde{\pi}(\tau+k h))$ and $\Pi^{0}=u(\tilde{\pi}(\tau))$ and get

$$
\begin{aligned}
\mathcal{I}_{\Pi}= & -\int_{\Omega^{k}} n^{k}\left(S^{k}-S^{0}\right)\left(u^{k}-u^{0}\right) \mathrm{d} x-\int_{\Omega^{k}} n^{k} S^{0}\left(u^{k}-u^{0}\right) \mathrm{d} x \\
& -h \sum_{j=1}^{k} \int_{\Omega^{j-1}} \tilde{k}^{j} \nabla u^{j} \nabla\left(u^{k}-u^{0}\right) \mathrm{d} x+\int_{\Omega^{1}} n(\tau) S^{0}\left(u^{k}-u^{0}\right) \mathrm{d} x \\
& -\sum_{j=2}^{k} \int_{\Omega^{j-1} \backslash \Omega^{j}} S^{j-1} n^{j-1}\left(u^{k}-u^{0}\right) \mathrm{d} x \\
& -h \sum_{j=1}^{k} \sum_{i m l} \int_{\partial B_{i m l}^{j}}\left(u^{k}-u^{0}\right) n^{j} f\left(R_{\max }-R_{i m l}^{j}\right) \\
& \times\left\{\left(S\left(u^{j}\right)-S_{0}\right)_{+}-S\left(u^{j}\right) \int_{\partial B_{i m l}^{j}} n^{j}\left(S\left(u^{j}\right)-S_{0}\right)_{+}\right\} \mathrm{d} \sigma,
\end{aligned}
$$

where $\mathcal{I}_{\Pi}$ is the term analogous to the right-hand side but with $u^{k}-u^{0}$ replaced by $\Pi^{k}-\Pi^{0}$. 
After integration between 0 and $T-k h$, with respect to $\tau$, we obtain

$$
\begin{aligned}
& \int_{0}^{T-k h} \mathcal{I}_{\Pi} \mathrm{d} \tau+\int_{0}^{T-k h} \int_{\Omega^{k}} n^{k}(S(\tau+k h)-S(\tau))(u(\tau+k h)-u(\tau)) \mathrm{d} x \mathrm{~d} \tau \\
& \leqslant C\left\{\int_{0}^{T-k h} \int_{\Omega^{k}}|n(\tau+k h)-n(\tau)|\left|u^{k}-u^{0}\right| \mathrm{d} x \mathrm{~d} \tau\right. \\
& +h \sum_{j=1}^{k} \int_{0}^{T-k h} \int_{\Omega^{j}}\left|\tilde{k}^{j} \nabla u^{j} \nabla\left(u^{k}-u^{0}\right)\right| \mathrm{d} x \mathrm{~d} \tau+\sum_{j=2}^{k} \int_{0}^{T-k h} \int_{\Omega^{j-1} \backslash \Omega^{j}}\left|u^{k}-u^{0}\right| \mathrm{d} x \mathrm{~d} \tau \\
& \left.+C h \sum_{j=1}^{k} \int_{0}^{T-k h} \int_{\Omega^{j}}\left(\epsilon\left|\nabla\left(u^{k}-u^{0}\right)\right|+\left|u^{k}-u^{0}\right|\right) \mathrm{d} x \mathrm{~d} \tau\right\} \\
& \leqslant C\left(\int_{0}^{T-k h} \int_{\Omega^{k}}|n(\tau+k h)-n(\tau)|^{2} \mathrm{~d} x \mathrm{~d} \tau\right)^{1 / 2} \\
& +C k h+C\left(\int_{0}^{T-k h} \int_{\Omega^{1} \backslash \Omega^{k}} \mathrm{~d} x \mathrm{~d} \tau\right)^{5 / 6},
\end{aligned}
$$

where we have used the embedding of $H^{1}$ in $L^{6}$. Let us now estimate the integrals at the right-hand side of (6.3).

First, by using the equation for $R_{i m l}^{j}$ we get

$$
\begin{aligned}
& \int_{0}^{T-k h} \int_{\Omega^{1} \backslash \Omega^{k}} \mathrm{~d} x \mathrm{~d} t=4 \pi h \int_{0}^{T-k h} \sum_{j=2}^{k} \sum_{i m l} f\left(R_{\max }-R_{i m l}^{j}\right) \\
& \times\left(R_{i m l}^{j}\right)^{2} \int_{\partial B_{i m k}^{j}} n^{j}\left(S\left(u^{j}\right)-S_{0}\right)_{+} \mathrm{d} \sigma \mathrm{d} \tau \leqslant C k h .
\end{aligned}
$$

Since the porosity $n$ is a Lipschitzian function satisfying an ODE, we have

$$
|n(\tau+k h)-n(\tau)| \leqslant C k h
$$

with a constant $C$ independent of $\epsilon$.

Completely analogous considerations lead to

$$
\left|\int_{0}^{T-k h} \mathcal{I}_{\Pi} \mathrm{d} \tau\right| \leqslant C k h
$$

Consequently, (6.3) reduces to

$$
\int_{0}^{T-k h} \int_{\Omega^{k}} n^{k}(S(\tau+k h)-S(\tau))(u(\tau+k h)-u(\tau)) \mathrm{d} x \mathrm{~d} \tau \leqslant C(k h)^{5 / 6} .
$$

Since $u_{h}$ is a step function in time, we see that this estimate is also satisfied if we replace $k h$ by any positive number $\eta$, i.e. we have

$$
\begin{aligned}
& \int_{0}^{T-\eta} \int_{\Omega(\tau+\eta)} n(\tau+\eta)\left(S\left(u_{h N}(\tau+\eta)\right)-S\left(u_{h N}(\tau)\right)\right) \\
& \quad \times(u(\tau+\eta)-u(\tau)) \mathrm{d} x \mathrm{~d} \tau \leqslant C \eta^{5 / 6}, \quad \eta>0 .
\end{aligned}
$$


We note that $S \in C^{0, \chi /(1+\beta \chi)}$ and following [9] from (6.7) we obtain

$$
\int_{0}^{T-\eta} \int_{\Omega(\tau+\eta)}\left|S\left(u_{h}(\tau+\eta)\right)-S\left(u_{h}(\tau)\right)\right| \mathrm{d} x \mathrm{~d} \tau \leqslant C \eta^{\frac{5 \chi}{6(1+\chi(1+\beta))}}
$$

and the proposition is proved.

On the basis of the previous estimate we can draw the following conclusion.

THEOREM 2 There is a subsequence of $\left\{u_{h}\right\}$, denoted by the same symbol, and a non-negative function $u \in L^{2}\left(0, T ; H^{1}(\Omega)\right)$ such that

$$
u_{h} \rightarrow u \text { weakly in } L^{2}\left(0, T ; H^{1}(\Omega)\right)
$$

and

$$
S\left(u_{h}\right) \rightarrow S(u) \text { strongly in } L^{1}(\Omega \times(0, T)), \text { as } h \rightarrow 0 .
$$

Proof. (6.9) follows immediately. (6.10) comes from monotonicity and the estimate (6.8). For more details we refer to [2] or [9].

\section{Existence of a weak solution}

In this section we are revisiting the passing to the limit for the time discretized problem. Classically, the a priori estimates obtained in the preceding sections imply the strong convergences and passing to the limit is an exercise. Nevertheless, we have to limit integrals over a domain which changes in time as a function of the solution. Then passing to the limit is not clear at all and we are obliged to give a proof which present a novelty in the theory of the degenerate non-linear parabolic equations.

Let $\lambda=\frac{\chi}{1+\beta \chi}$. Then $S \in C^{0, \lambda}$ as a function of $u$. We note that $W^{s, q}(\Omega(t)), 0<s<1$ and $1<q<+\infty$, is a Sobolev space of a fractional order. For its definition we refer to the book [16]. Then if $\varphi \in H^{1}(\Omega(t)), S(\varphi) \in W^{\lambda, 2 / \lambda}(\Omega(t))$. A simple interpolation argument and Theorem 2 imply

$$
S\left(u_{h}\right) \rightarrow S(u) \text { in } L^{2}\left(0, T ; W^{s, q}(\Omega(t)), 0<s<\lambda, 1<q<2 / \lambda .\right.
$$

Next we make use of a trace theorem from the book [3]. Since it is always possible to choose $s \in(\lambda / 2, \lambda)$ and $q \in(1,2 / \lambda)$ such that $s-1 / q>0$, we use the fact that the zero-order trace of a function from $W^{s, q}(\Omega(t))$ is in $W^{s-1 / q, q}(\partial B(t))$, where $B(t)$ is an arbitrary growing ball, and get

$$
S\left(u_{h}\right) \rightarrow S(u) \quad \text { in } L^{2}\left(0, T ; H^{b}(\partial B(t))\right), \quad b<\frac{\lambda}{2} .
$$

Now we write the discretized problem in the form which corresponds to the weak form of the continuous problem. 
First, we observe that

$$
\begin{aligned}
& \sum_{j=1}^{k} \int_{\Omega^{j}} n^{j} \partial_{t}^{-h} S\left(u^{j}\right) \varphi^{j} \mathrm{~d} x=-\sum_{j=1}^{k} \int_{\Omega^{j}} n^{j-1} S\left(u^{j-1}\right) \partial_{t}^{-h} \varphi^{j} \mathrm{~d} x \\
& +\sum_{j=0}^{k-1} \int_{\Omega^{j+1}} \frac{n^{j}-n^{j+1}}{h} S\left(u^{j}\right) \varphi^{j+1} \mathrm{~d} x \\
& +\int_{\Omega^{k}} \frac{n^{k}}{h} S\left(u^{k}\right) \varphi^{k} \mathrm{~d} x-\int_{\Omega^{1}} \frac{n^{0} S\left(u^{0}\right) \varphi^{0}}{h} \mathrm{~d} x \\
& +\sum_{j=1}^{k-1} \int_{\Omega^{j} \backslash \Omega^{j+1}} \frac{n^{j}}{h} S\left(u^{j}\right) \varphi^{j} \mathrm{~d} x
\end{aligned}
$$

where $\varphi^{j}$ is a linear combination of products of $\varphi_{m}(x)$ and $\psi_{l}(t), \varphi_{m} \in V$ and $\psi_{l} \in C^{\infty}([0, T])$. The index $j$ labels the interval $((j-1) h, j h]$.

Now the discretized weak formulation is

$$
\begin{aligned}
& -\sum_{j=1}^{k} h \int_{\Omega^{j}} n^{j-1} S\left(u^{j-1}\right) \partial_{t}^{-h} \varphi^{j} \mathrm{~d} x+\int_{\Omega^{k}} n^{k} S\left(u^{k}\right) \varphi^{k} \mathrm{~d} x \\
& -\int_{\Omega(0)} n^{0} S\left(u^{0}\right)+\sum_{j=1} \int_{\Omega^{j} \backslash \Omega^{j+1}} n^{j} S\left(u^{j}\right) \varphi^{j} \mathrm{~d} x \\
& +\sum_{j=1}^{k} h \int_{\Omega^{j-1}} \tilde{k}^{j} \nabla u^{j} \nabla \varphi^{j} \mathrm{~d} x \\
& +h \sum_{j=1}^{k} \sum_{i m l} \int_{\partial B_{i m l}^{j}} \varphi^{j} n^{j} f\left(R_{\max }-R_{i m l}^{j}\right)\left\{\left(S\left(u^{j}\right)-S_{0}\right)_{+}\right. \\
& \left.-S\left(u^{j}\right) \int_{\partial B_{i m l}^{j}} n^{j}\left(S\left(u^{j}\right)-S_{0}\right)_{+} \mathrm{d} \sigma\right\}=0 .
\end{aligned}
$$

In order to study convergence of the various terms we start with establishing convergence for the radii $R_{i m l}^{(h)}(t)=R_{i m k}^{j}$ for $t \in((j-1) h, j h]$. Using (4.5) we get the following lemma.

Lemma 4 There exists $R_{i m l} \in C^{0,1}([0, T])$ and a subsequence of $\left\{R_{i m l}^{(h)}\right\}$ denoted by the same symbol, such that

$$
\begin{aligned}
& R_{i m l}^{(h)} \rightarrow R_{i m l} \quad \text { in } L^{\infty}(0, T) \\
& \partial_{t}^{-h} R_{i m l}^{(h)} \rightarrow \partial_{t} R_{i m l} \geqslant 0 \quad \text { weak-* in } L^{\infty}(0, T) \\
& \left\|R_{i m l}^{(h)}-R_{i m l}\right\|_{L^{\infty}(0, T)} \leqslant C h .
\end{aligned}
$$

We note that $n^{(h)}(x, t)=n^{j}(x)$ for $t \in((j-1) h, j h]$. Then $n^{(h)}$ is bounded from below by a positive constant independent of $h$ and we have the following corollary. 
COROLLARY 1 There is a subsequence of $\left\{n^{(h)}\right\}$, denoted by the same symbol, and $n \in$ $C^{0,1}(\overline{\Omega(0)} \times[0, T])$ such that

$$
\begin{aligned}
& n^{(h)} \rightarrow n \quad \text { in } L^{\infty}(0, T ; C(\overline{\Omega(0)})) \\
& \partial_{t}^{h} n^{(h)} \stackrel{*}{\rightarrow} \partial_{t} n \quad \text { weak-* in } L^{\infty}(C(\overline{\Omega(0)} \times(0, T))
\end{aligned}
$$

and $n$ is linked with $R_{i m l}$ by (2.6).

Now we are ready to limit the absorption terms.

Proposition 2 Let $\partial B_{i m l}^{h}(t)=\partial B_{i m l}^{j}$ for $t \in((j-1) h, j h]$. Then we have

$$
\begin{gathered}
\int_{\partial B_{i m l}^{h}(t)} n^{h}\left(S\left(u_{h}\right)-S_{0}\right)_{+} \mathrm{d} \sigma \rightarrow \int_{\partial B_{i m l}(t)} n\left(S(u)-S_{0}\right)_{+} \mathrm{d} \sigma \\
\text { in } \quad L^{2}(0, T)
\end{gathered}
$$

when $h \rightarrow 0$, where $\partial B_{i m l}(t)=\left\{y|| y-r_{i m l} \mid=R_{i m l}(t)\right\}$.

In order to prove Proposition 2 we prove the following auxiliary result

LeMma 5 Let $B_{i m l}$ be a ball with center at $r_{i m l}$ and let us fix a local spherical coordinate system with 0 at $r_{i m l}$. Let $0<a<b, b-a=h$ and let $z \in H^{1}(\Omega)$. Then for arbitrary $\eta \in[a, b]$ we have

$$
\begin{aligned}
& \int_{\{\text {angles\} }}\left|\frac{1}{h} \int_{a}^{b} \xi^{2} S(z(\xi, \alpha)) \mathrm{d} \xi-\frac{b^{3}-a^{3}}{3 h} S(z(\eta, \alpha))\right| \mathrm{d} \mathcal{A} \\
\leqslant & C h^{\lambda / 2}\|z\|_{H^{1}(] a, b[\times\{r=1\})}
\end{aligned}
$$

where $\lambda=\chi /(1+\beta \chi), S \in C^{0, \lambda}$ and $\{r=1\}$ denotes the surface of the unit ball. Finally, $C=C(b, a)$ and $\mathrm{d} \mathcal{A}=\sin \vartheta \mathrm{d} \vartheta \mathrm{d} \varphi$ is the surface measure of the unit sphere.

Proof. Since $S \in C^{0, \lambda}$ we have

$$
\begin{aligned}
I & =\int_{\text {\{angles\} }}\left|\frac{1}{h} \int_{a}^{b} \xi^{2} S(z(\xi, \alpha)) \mathrm{d} \xi-\frac{b^{3}-a^{3}}{3 h} S(z(\eta, \alpha))\right| \mathrm{d} \mathcal{A} \\
& \leqslant \frac{C}{h} \int_{\text {\{angles\} }} \int_{a}^{b} \xi^{2}|z(\xi, \alpha)-z(\eta, \alpha)|^{\lambda} \mathrm{d} \xi \mathrm{d} \mathcal{A} \\
& \leqslant \frac{C}{h} \int_{\text {\{angles\} }} \int_{a}^{b} \xi^{2}\left|\int_{\eta}^{\xi} \frac{\partial z}{\partial r}(r, \alpha) \frac{\mathrm{d} r}{|\xi-\eta|^{1 / 2}}\right|^{\lambda}|\xi-\eta|^{\lambda / 2} \mathrm{~d} \xi \mathrm{d} \mathcal{A} .
\end{aligned}
$$

By simple application of Hölder's inequality and using that

$$
\left(\int_{\{\text {angles\} }} \int_{a}^{b} \frac{\xi^{2}}{h}|\xi-\eta|^{\lambda /(2-\lambda)} \mathrm{d} \xi \mathrm{d} \mathcal{A}\right)^{1-\lambda / 2} \leqslant C\left(\frac{b}{a}\right) a^{2-\lambda} h^{\lambda / 2}
$$

we obtain

$$
\begin{aligned}
& I \leqslant C\left(\frac{b}{a}\right) a^{2-\lambda} h^{\lambda / 2}\left(\int_{\text {\{angles\} }} \int_{a}^{b}\left(\int_{\eta}^{\xi} \frac{\partial z}{\partial r}(r, \alpha) d r\right)^{2} \frac{\xi^{2}}{h|\xi-\eta|} \mathrm{d} \xi \mathrm{d} \mathcal{A}\right)^{\lambda / 2} \\
& \leqslant \bar{C}\left(\frac{b}{a}\right) a^{2-\lambda} h^{\lambda / 2}\left(\int_{\{\text {angles\} }} \int_{a}^{b} \frac{\sup \{b-\eta, \eta-\alpha\}}{h}\left|\frac{\partial z}{\partial r}(r, \alpha)\right|^{2} r^{2} \mathrm{~d} r \mathrm{~d} \mathcal{A}\right)^{\lambda / 2} \\
& \leqslant \bar{C}\left(\frac{b}{a}\right) a^{2-\lambda}\|z\|_{H^{1}(a, b[\times\{r=1\})} h^{\lambda / 2}
\end{aligned}
$$


and the lemma is proved.

Proof of Proposition 2. We write the boundary integral as

$$
\int_{\partial B_{i m l}^{h}(t)} n^{h}\left(S\left(u_{h}\right)-S_{0}\right)_{+} \mathrm{d} \sigma=\int_{\{\text {angles }\}}\left(R_{i m l}^{(h)}(t)\right)^{2} n^{h}\left(S\left(u_{h}\left(R_{i m l}^{(h)}(t), \alpha\right)\right)-S_{0}\right)_{+} \mathrm{d} \mathcal{A}
$$

where we use the local spherical coordinates with center at $r_{i m l}$.

Since

$$
\begin{aligned}
& \left|\int_{\{\text {angles\} }} n^{h}\left(\left(R_{i m l}^{(h)}(t)\right)^{2}-R_{i m l}^{2}(t)\right)\left(S\left(u_{h}\left(R_{i m l}^{(h)}(t), \alpha\right)\right)-S_{0}\right)_{+} \mathrm{d} \mathcal{A}\right| \\
\leqslant & C\left|R_{i m l}^{(h)}(t)-R_{i m l}(t)\right|
\end{aligned}
$$

and, by Lemma 5 with $\eta=R_{i m l}^{(h)}$ and $R_{i m l}$ respectively,

$$
\left|\int_{\text {\{angles\} }} R_{i m l}^{2}(t)\left(\left(S\left(u_{h}\left(R_{i m l}^{(h)}(t), \alpha\right)\right)-S_{0}\right)_{+}-\left(S\left(u_{h}\left(R_{i m l}(t), \alpha\right)\right)-S_{0}\right)_{+}\right)\right| \leqslant C h^{\lambda / 2} .
$$

Then the limit of the expression in (7.13) is equal to the limit of

$$
\left.\int_{\text {\{angles\} }} R_{i m l}^{2}(t)\left(S\left(u_{h}\left(R_{i m l}(t), \alpha\right)\right)-S_{0}\right)_{+} \mathrm{d} \mathcal{A}=\int_{\partial B_{i m l}(t)} n^{h}\left(S\left(u_{h}\right)-S_{0}\right)_{+}\right) \mathrm{d} \sigma
$$

Now the convergence (7.2) implies (7.9).

COROLlary 2 We have

$$
\partial_{t}^{-h} R_{i m l}^{h}(t) \rightarrow \partial_{t} R_{i m l} \quad \text { in } L^{q}(0, T), \quad \forall q<+\infty,
$$

and

$$
4 \pi R_{i m l}^{2}(t) \frac{\mathrm{d}}{\mathrm{d} t} R_{i m l}(t)=f\left(R_{\max }-R_{i m l}(t)\right) \int_{\partial B_{i m l}(t)} n\left(S(u)-S_{0}\right)_{+} \mathrm{d} \sigma
$$

where $B_{i m l}(t)=\left\{x|| x-r_{i m l} \mid<R_{i m l}(t)\right\}$ and $u$ is defined by (6.9)-(6.10).

Limiting the terms in the discretized weak formulation (7.3) is now straightforward.

COROLlary 3

$$
\begin{aligned}
& \lim _{h \rightarrow 0} h \sum_{j=1}^{k} \sum_{i m l} \int_{\partial B_{i m l}^{j}} n^{j} \varphi^{j} f\left(R_{\max }-R_{i m l}^{j}\right) \\
& \times\left\{\left(S\left(u^{j}\right)-S_{0}\right)_{+}-S\left(u^{j}\right) \int_{\partial B_{i m l}^{j}} n^{j}\left(S\left(u^{j}\right)-S_{0}\right)_{+} \mathrm{d} \sigma\right\} \mathrm{d} \sigma \\
= & \int_{0}^{T} \sum_{i m l} n^{j} f\left(R_{\max }-R_{i m l}(t)\right) \int_{\partial B_{i m l}(t)} \varphi\left\{\left(S(u)-S_{0}\right)_{+}\right. \\
& \left.-S(u) \int_{\partial B_{i m l}(t)} n^{j}\left(S(u)-S_{0}\right)_{+} \mathrm{d} \sigma\right\} \mathrm{d} \sigma .
\end{aligned}
$$


LEMMA 6

$$
\begin{aligned}
& \lim _{h \rightarrow 0} h \sum_{j=0}^{k-1} \int_{\Omega^{j} \backslash \Omega^{j+1}} \frac{1}{h} n^{j} S\left(u^{j}\right) \varphi^{j} \mathrm{~d} x \\
= & \int_{0}^{T} \int_{\partial \Omega(t) \backslash \partial \Omega} n S(u) \varphi R^{2}(t) \frac{\mathrm{d} R}{\mathrm{~d} t} \mathrm{~d} \sigma \mathrm{d} t, \quad \forall \varphi \in C^{\infty}(\bar{\Omega} \times[0, T])
\end{aligned}
$$

where $R(t)=R_{i m l}(t)$ for the ball $B_{i m l}(t)$.

Proof. We have

$$
\begin{aligned}
I_{h} & =\sum_{j=0}^{k-1} \int_{\Omega^{j} \backslash \Omega^{j+1}} \frac{1}{h} n^{j} S\left(u_{h}\right) \varphi^{j} \mathrm{~d} x \\
& =\sum_{m n l} \sum_{j=0}^{k-1} \int_{j h}^{(j+1) h} \int_{\{\text {angles\} }} \frac{1}{h} \int_{R_{m n l}^{j}}^{R_{m n l}^{j+1}} n^{j} S\left(u_{h}\right) \varphi r^{2} \mathrm{~d} \mathcal{A} \mathrm{d} t .
\end{aligned}
$$

Then by Lemma 5

$$
\begin{aligned}
& \left|I_{h}-\sum_{m n l} \sum_{j=0}^{k-1} \int_{j h}^{(j+1) h} \int_{\{\text {angles }\}} \frac{\left(R_{m n l}^{j+1}\right)^{3}-\left(R_{m n l}^{j}\right)^{3}}{3 h} n^{j} S\left(u_{h}\left(R_{m n l}(t), \alpha\right)\right) \varphi \mathrm{d} \mathcal{A} \mathrm{d} t\right| \\
& \leqslant C h^{\lambda / 2}\left\|u_{h}\right\|_{L^{2}\left(0, T ; H^{1}(\Omega)\right)} \cdot\|\varphi\|_{L^{\infty}\left(Q_{T}\right)} .
\end{aligned}
$$

Therefore

$$
\begin{aligned}
\lim _{h \rightarrow 0} I_{h} & =\lim _{h \rightarrow 0} \sum_{m n l} h \sum_{j=0}^{k-1} \frac{1}{h} \int_{j h}^{(j+1) h} \int_{\{\text {angles }\}} \frac{\left(R_{m n l}^{j+1}\right)^{3}-\left(R_{m n l}^{j}\right)^{3}}{3 h} n^{j} S\left(u_{h}\left(R_{m n l}(t), \alpha\right)\right) \varphi \mathrm{d} \mathcal{A} \mathrm{d} t \\
& =\sum_{m n l} \int_{0}^{T} \int_{\partial B_{m n l}(t)} n S(u) \varphi R^{2}(t) \frac{\mathrm{d} R}{\mathrm{~d} t} \mathrm{~d} \sigma \mathrm{d} t,
\end{aligned}
$$

and the lemma is proved.

LEMMA 7

$$
\begin{aligned}
& \lim _{h \rightarrow 0} \sum_{j=1}^{k} h \int_{\Omega^{j-1}} \tilde{k}^{j} \nabla u^{j} \nabla \varphi^{j} \mathrm{~d} x \\
= & \int_{0}^{T} \int_{\partial \Omega(t)} \tilde{k} \nabla u \nabla \varphi \mathrm{d} x \mathrm{~d} t, \quad \forall \varphi \in C^{\infty}(\bar{\Omega} \times[0, T]) .
\end{aligned}
$$

Proof. We have

$$
\begin{aligned}
& h \sum_{j=1}^{k} \int_{\Omega^{j-1}} \tilde{k}^{j} \nabla u^{j} \nabla \varphi^{j} \mathrm{~d} x \\
= & \int_{0}^{T} \int_{\Omega(t)} \widetilde{k}^{h} \nabla u_{h} \nabla \varphi \mathrm{d} x \mathrm{~d} t+\sum_{j=1}^{k} \int_{j h}^{(j+1) h} \int_{\Omega^{j-1} \backslash \Omega(t)} \tilde{k}^{j} \nabla u^{j} \nabla \varphi^{j} \mathrm{~d} x \mathrm{~d} t .
\end{aligned}
$$


Then

$$
\lim _{h \rightarrow 0} \int_{0}^{T} \int_{\Omega(t)} \widetilde{k}^{h} \nabla u_{h} \nabla \varphi \mathrm{d} x \mathrm{~d} t=\int_{0}^{T} \int_{\Omega(t)} \tilde{k} \nabla u \nabla \varphi \mathrm{d} x \mathrm{~d} t
$$

and

$$
\begin{aligned}
& \left|\sum_{j=1}^{k} \int_{j h}^{(j+1) h} \int_{\Omega^{j} \backslash \Omega(t)} \tilde{k}^{j} \nabla u^{j} \nabla \varphi^{j} \mathrm{~d} x\right| \\
\leqslant & C\left\|u_{h}\right\|_{L^{2}\left(0, T ; H^{1}(\Omega)\right)} \cdot\left(\int_{0}^{T}\left|\Omega^{h}(t) \backslash \Omega(t)\right| \mathrm{d} t\right)^{1 / 2}\|\varphi\|_{C^{0,1}\left(Q_{T}\right)} .
\end{aligned}
$$

As

$$
\left|\Omega^{h}(t) \backslash \Omega(t)\right| \leqslant C \sum_{m n l}\left|R_{m n l}^{(h)}(t)-R_{m n l}(t)\right| \rightarrow 0,
$$

(7.20) and (7.21) imply (7.19).

Now we are able to state our result.

THEOREM 3 There is a function $u \in L^{2}\left(0, T ; H^{1}(\Omega)\right), u \geqslant 0$ a.e. on $Q_{T}$,

$$
\int_{0}^{r-\eta} \int_{\Omega(\tau+\eta)}|S(u(\tau+\eta))-S(u(\tau))| \mathrm{d} x \mathrm{~d} t \leqslant C \eta^{\beta},
$$

for some $\beta>0$, and $R_{i m k} \in C^{0,1}([0, T])$ such that (3.4) and (3.6) hold true, for every $\varphi \in$ $H^{1}(\Omega \times(0, T)), \varphi(x, T)=0$ and $\left.\varphi\right|_{x_{1}=0}=0$, i.e. there is a weak solution for the problem (P).

Proof. It is a simple consequence of the preceding results.

REMARK 4 Since $u \geqslant 0$ a.e. it is possible to reconstruct the pressure field $p$. On the sets $\{u(x, t) \geqslant$ $c>0\}$ we use (3.2) to calculate $p(x, t)$. On the set $\{u(x, t)=0\}$ we know only that $p \leqslant 0$ but we don't have more information.

\section{Acknowledgements}

This work has been partially supported by the Italian MURST Project on Free Boundary Problems.

\section{REFERENCES}

1. Alt, H. W. \& DiBenedetto, E. Nonsteady flow of water and oil through inhomogeneous porous media. Ann. Scuola Norm. Sup. Pisa 12, (1985) 335-392.

2. Alt, H. W. \& LuCKhaus, S. Quasilinear elliptic-parabolic equations. Math. Z. 183, (1983) 311-341.

3. BERGH, J. \& LÖFSTRÖM, J. Interpolation Spaces, an Introduction. Springer, Berlin (1976).

4. CARrillo, J. Entropy solutions for nonlinear degenerate problems. Arch. Rational Mech. Anal. 147, (1999) 269-361.

5. Cioranescu, D. \& Saint Jean Paulin, J. Homogenization in open sets with holes. J. Math. Anal. Appl. 71, (1979) 590-607.

6. Comparini, E., GiAnni, R., \& MANnUCCI, P. A filtration problem in a composite porous material with two free boundaries. To appear in Adv. Math. Sci. Appl. 11, (2001). 
7. FASAnO, A. Porous media with hydrophile granules. In: FASANO, A. (ed), Complex Flows in Industrial Processes, Chapter 10. pp. 307-332. MSSET Birkhäuser, Boston, MA (2000).

8. FASAno, A. A one dimensional flow problem in porous media with hydrophile grains. Math. Meth. Appl. Sci. 22, (1999) 605-617.

9. FASANO, A. \& Mikelić, A. On the filtration through porous media with partially soluble permeable grains. Nonlin. Diff. Eq. Appl. 7, (2000) 91-105.

10. Fasano, A. \& Solonnikov, V. On one-dimensional unsaturated medium with hydrophile grains. Funct. Diff. Eq. 8, (2001) 195-224.

11. GiAnni, R. \& MANNUCCI, P. The propagation of a wetting front through an absorbing porous material with saturation dependent permeability. Nonlin. Diff. Eq. Appl. 8, (2001) 219-235.

12. GiUsti, E. Minimal Surfaces and Functions of Bounded Variation. Birkhäuser, Boston, MA (1984).

13. Jikov, V. V., Kozlov, S. M., \& Oleinik, O. A. Homogenization of Differential Operators and Integral Functionals. Springer, Berlin (1994).

14. KRÖNer, D. \& LuCKhaUs, S. Flow of oil and water in a porous medium. J. Diff. Eqns 55, (1984) 276-288.

15. Отто, F. $L^{1}$-contractions and uniqueness for quasilinear elliptic-parabolic equations. J. Diff. Eqns 131, (1996) 20-38.

16. TRIeBel, H. Interpolation Theory, Function Spaces, Differential Operators. VEB Deutschen, Berlin (1978). 